\title{
An Estimator of the Mean Estimation of Study Variable Using Median of Auxiliary Variable
}

\author{
Sunil Kumar* \\ Alliance University, Bangalore, India \\ *sunilbhougal06@gmail.com
}

Received: 07, October 2014/ Revised: 26, June 2015 / Accepted: 24, August 2015

CIAppStat-SL2015

\begin{abstract}
In the present study, I propose a modified estimator for estimating the population mean of the study variable auxiliary information when the population mean and the population median of the auxiliary variable is known. The expression of bias and mean squared error (MSE) of the proposed estimator is derived. Some existing estimators are also discussed. Comparisons of the proposed estimator with the other estimators are carried out. The results obtained are illustrated numerically by using three natural populations considered in the literature.
\end{abstract}

Keywords: Mean squared error, bias, median, simple random sampling

\section{Introduction}

\subsection{Introduction to the Research Problem}

Use of auxiliary information in the estimation of population parameters such as population mean, ratio of two population means, product of two population means, coefficient of variation etc. has been in practice. Ratio, product and regression type estimators are good examples in this context. Cochran (1940) initiated the use of auxiliary information at estimation stage and proposed ratio estimator for population mean. It is well established fact that ratio type estimators provide better efficiency in comparison to simple mean estimator if the study variable and auxiliary variable are positively correlated. If the correlation between the study variable and auxiliary variables negative, product estimator given by Robson (1957) is more efficient than simple mean estimator.

Further improvements are also achieved on the classical ratio estimator by introducing a large number of modified ratio estimators with the use of known parameters like, coefficient of variation, coefficient of kurtosis, coefficient of 
skewness and population correlation coefficient. For more detailed discussion one may refer to Cochran (1977), Kadilar and Cingi (2004, 2006), Koyuncu and Kadilar (2009), Murthy (1967), Prasad (1989), Rao (1991), Singh (2003), Singh and Tailor (2003, 2005), Singh et al (2004), Sisodia and Dwivedi (1981), Upadhyaya and Singh (1999) and Yan and Tian (2010).

Further, Subramani and Kumarapandiyan (2013) had taken initiative by proposed modified ratio estimator for estimating the population mean of the study variable by using the population median of the auxiliary variable.

The objective of the paper is to proposed modified estimator for estimating the population mean by using the population median of the auxiliary variable.

\section{Notations Used}

The following are the notations used in the paper:

$N$ : Size of the finite population; $n$ : Sample size taken from the population of size $N ; y$ : Study variable; $x$ : Auxiliary variable; $y_{i}$ : Value of the $i^{\text {th }}$ unit of the sample for the study variable $y ; x_{i}$ : Value of the $i^{t h}$ unit of the sample for the auxiliary variable $x ; \bar{Y}=\sum_{i=1}^{N} y_{i} / N$ : Population mean for study variable $y$ for the entire population; $\bar{X}=\sum_{i=1}^{N} x_{i} / N$ : Population mean for the auxiliary variable $x$ for the entire population; $\bar{y}=\sum_{i=1}^{n} y_{i} / n$ : Sample mean for the study variable $y$ for the sample of size $n ; \bar{x}=\sum_{i=1}^{n} x_{i} / n$ : Sample mean for the auxiliary variable $x$ for the sample of size $n ; S_{y}^{2}=\sum_{i=1}^{N}\left(y_{i}-\bar{Y}\right)^{2} /(N-1)$ : Population mean square of the study variable $y$ for the entire population; $S_{x}^{2}=\sum_{i=1}^{N}\left(x_{i}-\bar{X}\right)^{2} /(N-1)$ : Population mean square of the auxiliary variable $x$ for the entire population; $S_{x y}=\sum_{i=1}^{N}\left(y_{i}-\bar{Y}\right)\left(x_{i}-\bar{X}\right) /(N-1)$ : Population covariance between $y$ and $x$ for the entire population; $\rho_{y x}=\frac{s_{x y}}{s_{x} S_{y}}$ : Correlation coefficient between $y$ and $x$ in the entire population; $C_{y}=S_{y} / \bar{Y}$ : Coefficient of variation $(\mathrm{CV})$ of the study variable $y$ in the entire population; $C_{x}=S_{x} / \bar{X}$ : Coefficient of variation $(\mathrm{CV})$ of the auxiliary variable $x$ in the entire population;,$\beta_{1}=\frac{N \sum_{i=1}^{N}\left(X_{i}-\bar{X}\right)^{3}}{(N-1)(N-2) S^{3}}$ is the coefficient of Skewness of the auxiliary variable, $\beta_{2}=\frac{N(N+1) \sum_{i=1}^{N}\left(X_{i}-\bar{X}\right)^{4}}{(N-1)(N-2)(N-3) S^{4}}-\frac{3(N-1)^{2}}{(N-2)(N-3)}$ is the coefficient of Kurtosis of the auxiliary variable, and $M_{d}$ is the population median of the auxiliary variable.

\section{Procedure and Definitions}

Consider a finite population $U=\left(U_{1}, U_{2}, \ldots, U_{N}\right)$ of size $N$. Let $y$ and $x$ denote the study variable and the auxiliary variable taking values $y_{i}$ and $x_{i}$ respectively on the $\mathrm{i}^{\text {th }}$ unit $(\mathrm{i}=1,2, \ldots, \mathrm{N})$. For estimating the population mean $\bar{Y}$ of $y$ a simple random 
sample of size $n$ is draw without replacement from the population $U$. Then the classical ratio estimator is defined as

$$
t_{R}=\frac{\bar{y}}{\bar{x}} \bar{X} ; \text { if } \bar{x} \neq 0
$$

and the product estimator is given by

$$
t_{P}=\frac{\bar{y}}{\bar{X}} \bar{x}
$$

where $\bar{X}$, the population mean of the auxiliary variable $\mathrm{x}$ is known.

The mean squared error expressions of the ratio and product estimators are

$$
\begin{aligned}
& \operatorname{MSE}\left(t_{R}\right)=\left(\frac{1-f}{n}\right) \bar{Y}^{2}\left\{C_{y}^{2}+C_{x}^{2}\left(1-2 \rho_{y x} \frac{C_{y}}{C_{x}}\right)\right\} \\
& \operatorname{MSE}\left(t_{P}\right)=\left(\frac{1-f}{n}\right) \bar{Y}^{2}\left\{C_{y}^{2}+C_{x}^{2}\left(1+2 \rho_{y x} \frac{C_{y}}{C_{x}}\right)\right\}
\end{aligned}
$$

Further, a list of modified ratio estimators is given in table 1 is used for assessing the performance of the proposed estimator along with their bias and mean squared error

\begin{tabular}{|c|c|c|c|}
\hline Estimator & Bias, B(.) & $\begin{array}{c}\text { Mean squared } \\
\text { error, MSE(.) }\end{array}$ & $\begin{array}{l}\text { Constants } \theta_{i} \\
\text { or } R_{i}\end{array}$ \\
\hline$t_{1}=\bar{y}\left(\frac{\bar{X}+C_{x}}{\bar{x}+C_{x}}\right)$ & $\left(\frac{1-f}{n}\right) \bar{Y}\left(\theta_{1}^{2} C_{x}^{2}\right.$ & $\left(\frac{1-f}{n}\right) \bar{Y}^{2}\left(C_{y}^{2}\right.$ & $\theta_{1}=\frac{\bar{X}}{\bar{X}+C_{x}}$ \\
\hline $\begin{array}{l}\text { Sisodia and Dwivedi } \\
(1981)\end{array}$ & $\left.-2 \theta_{1} C_{x} C_{y} \rho_{y x}\right)$ & $\begin{array}{l}+\theta_{1}^{2} C_{x}^{2} \\
\left.-2 \theta_{1} C_{x} C_{y} \rho_{y x}\right)\end{array}$ & \\
\hline$t_{2}=\bar{y}\left(\frac{\bar{X}+\beta_{2}}{\bar{x}+\beta_{2}}\right)$ & $\left(\frac{1-f}{n}\right) \bar{Y}\left(\theta_{2}^{2} C_{x}^{2}\right.$ & $\left(\frac{1-f}{n}\right) \bar{Y}^{2}\left(C_{y}^{2}\right.$ & $\theta_{2}=\frac{\bar{X}}{\bar{X}+\beta_{2}}$ \\
\hline Singh et al (2004) & $\left.-2 \theta_{2} C_{x} C_{y} \rho_{y x}\right)$ & $\begin{array}{l}+\theta_{2}^{2} C_{x}^{2} \\
\left.-2 \theta_{2} C_{x} C_{y} \rho_{y x}\right)\end{array}$ & \\
\hline$t_{3}=\bar{y}\left(\frac{\bar{X}+\beta_{1}}{\bar{x}+\beta_{1}}\right)$ & $\left(\frac{1-f}{n}\right) \bar{Y}\left(\theta_{3}^{2} C_{x}^{2}\right.$ & $\left(\frac{1-f}{n}\right) \bar{Y}^{2}\left(C_{y}^{2}\right.$ & $\theta_{3}=\frac{\bar{X}}{\bar{X}+\beta_{1}}$ \\
\hline Yan and Tian (2010) & $\left.-2 \theta_{3} C_{x} C_{y} \rho_{y x}\right)$ & $\begin{array}{l}+\theta_{3}^{2} C_{x}^{2} \\
\left.-2 \theta_{3} C_{x} C_{y} \rho_{y x}\right)\end{array}$ & \\
\hline$t_{4}=\bar{y}\left(\frac{\bar{X}+\rho_{y x}}{\overline{\bar{x}}+\rho}\right)$ & $\left(\frac{1-f}{n}\right) \bar{Y}\left(\theta_{4}^{2} C_{x}^{2}\right.$ & $\left(\frac{1-f}{n}\right) \bar{Y}^{2}\left(C_{y}^{2}\right.$ & $\theta_{4}=\frac{\bar{X}}{\overline{\bar{X}} \perp \Omega}$ \\
\hline Singh and Tailor (2003) & $\left.-2 \theta_{4} C_{x} C_{y} \rho_{y x}\right)$ & $\begin{array}{l}+\theta_{4}^{2} C_{x}^{2} \\
\left.-2 \theta_{4} C_{x} C_{y} \rho_{y x}\right)\end{array}$ & $\Lambda+\rho_{y x}$ \\
\hline$t_{5}=\bar{y}\left(\frac{\bar{X} C_{x}+\beta_{2}}{\bar{x} C_{x}+\beta_{2}}\right)$ & $\left(\frac{1-f}{n}\right) \bar{Y}\left(\theta_{5}^{2} C_{x}^{2}\right.$ & $\left(\frac{1-f}{n}\right) \bar{Y}^{2}\left(C_{y}^{2}\right.$ & $\begin{array}{l}\theta_{5} \\
=\bar{X} C_{x} \\
\end{array}$ \\
\hline $\begin{array}{l}\text { Upadhyaya and Singh } \\
\text { (1999) }\end{array}$ & $\left.-2 \theta_{5} C_{x} C_{y} \rho_{y x}\right)$ & $\begin{array}{l}+\theta_{5}^{2} C_{x}^{2} \\
\left.-2 \theta_{5} C_{x} C_{y} \rho_{y x}\right)\end{array}$ & $-\frac{\bar{X} C_{x}+\rho_{y x}}{2}$ \\
\hline $\begin{array}{c}\quad t_{6}=\frac{\bar{y}+b(\bar{X}-\bar{x})}{\bar{x}} \bar{X} \\
\text { Kadilar and Cingi (2004) }\end{array}$ & $\left(\frac{1-f}{n}\right) \frac{S_{x}^{2}}{\bar{Y}} R_{6}^{2}$ & $\begin{array}{l}\left(\frac{1-f}{n}\right)\left(R_{6}^{2} S_{x}^{2}\right. \\
\left.+S_{y}^{2}\left(1-\rho_{y x}^{2}\right)\right) \\
\end{array}$ & $R_{6}=\frac{\bar{Y}}{\bar{X}}$ \\
\hline
\end{tabular}
expressions.

Table 1: Existing modified ratio type estimators with their biases and mean squared errors 
Table 1 (continued):

\begin{tabular}{|c|c|c|c|}
\hline $\begin{array}{r}t_{7}=\frac{\bar{y}+b(\bar{X}-\bar{x})}{\bar{x}+C_{x}}(\bar{X} \\
\left.+C_{x}\right) \\
\text { Kadilar and Cingi }(2004) \\
t_{8}=\frac{\bar{y}+b(\bar{X}-\bar{x})}{\bar{x}+\beta_{2}}(\bar{X} \\
\left.+\beta_{2}\right)\end{array}$ & $\left(\frac{1-f}{n}\right) \frac{S_{x}^{2}}{\bar{Y}} R_{8}^{2}$ & $\begin{array}{l}\left(\frac{1-f}{n}\right)\left(R_{7}^{2} S_{x}^{2}\right. \\
\left.+S_{y}^{2}\left(1-\rho_{y x}^{2}\right)\right) \\
\left(\frac{1-f}{n}\right)\left(R_{8}^{2} S_{x}^{2}\right. \\
\left.+S_{y}^{2}\left(1-\rho_{y x}^{2}\right)\right)\end{array}$ & $\begin{array}{l}R_{7}=\frac{\bar{Y}}{\bar{X}+C_{x}} \\
R_{8}=\frac{\bar{Y}}{\bar{X}+\beta_{2}}\end{array}$ \\
\hline $\begin{array}{c}t_{9}=\frac{\bar{y}+b(\bar{X}-\bar{x})}{\bar{x} C_{x}+\beta_{2}}\left(\bar{X} C_{x}\right. \\
\left.+\beta_{2}\right)\end{array}$ & $\left(\frac{1-f}{n}\right) \frac{S_{x}^{2}}{\bar{Y}} R_{9}^{2}$ & $\begin{array}{l}\left(\frac{1-f}{n}\right)\left(R_{9}^{2} S_{x}^{2}\right. \\
\left.+S_{y}^{2}\left(1-\rho_{y x}^{2}\right)\right)\end{array}$ & $\begin{array}{l}R_{9} \\
=\frac{\bar{Y} C_{x}}{\bar{X} C_{x}+\beta_{2}}\end{array}$ \\
\hline $\begin{array}{c}t_{10}=\frac{\bar{y}+b(\bar{X}-\bar{x})}{\bar{x}+\beta_{1}}(\bar{X} \\
\left.+\beta_{1}\right) \\
\text { Yan and Tian }(2010)\end{array}$ & $\left(\frac{1-f}{n}\right) \frac{S_{x}^{2}}{\bar{Y}} R_{10}^{2}$ & $\begin{array}{l}\left(\frac{1-f}{n}\right)\left(R_{10}^{2} S_{x}^{2}\right. \\
\left.+S_{y}^{2}\left(1-\rho_{y x}^{2}\right)\right)\end{array}$ & $R_{10}=\frac{\bar{Y}}{\bar{X}+\beta_{1}}$ \\
\hline $\begin{array}{l}t_{11}=\frac{\bar{y}+b(\bar{X}-\bar{x})}{\bar{x} \beta_{1}+\beta_{2}}\left(\beta_{1} \bar{X}\right. \\
\left.+\beta_{2}\right) \\
\text { Yan and Tian }(2010)\end{array}$ & $\left(\frac{1-f}{n}\right) \frac{S_{x}^{2}}{\bar{Y}} R_{11}^{2}$ & $\begin{array}{l}\left(\frac{1-f}{n}\right)\left(R_{11}^{2} S_{x}^{2}\right. \\
\left.+S_{y}^{2}\left(1-\rho_{y x}^{2}\right)\right)\end{array}$ & $\begin{array}{l}R_{11} \\
=\frac{\bar{Y} \beta_{1}}{\bar{X} \beta_{1}+\beta_{2}}\end{array}$ \\
\hline $\begin{array}{r}t_{12}=\frac{\bar{y}+b(\bar{X}-\bar{x})}{\bar{x}+\rho_{y x}}(\bar{X} \\
\left.+\rho_{y x}\right)\end{array}$ & $\left(\frac{1-f}{n}\right) \frac{S_{x}^{2}}{\bar{Y}} R_{12}^{2}$ & $\begin{array}{l}\left(\frac{1-f}{n}\right)\left(R_{12}^{2} S_{x}^{2}\right. \\
\left.+S_{y}^{2}\left(1-\rho_{y x}^{2}\right)\right)\end{array}$ & $\begin{array}{l}R_{12} \\
=\frac{\bar{Y}}{\bar{X}+\rho_{y x}}\end{array}$ \\
\hline $\begin{array}{r}\begin{array}{r}t_{13}=\frac{\bar{y}+b(\bar{X}-\bar{x})}{\bar{x} C_{x}+\rho_{y x}}\left(\bar{X} C_{x}\right. \\
\left.+\rho_{y x}\right)\end{array} \\
\text { Kadilar and Cingi }(2006)\end{array}$ & $\left(\frac{1-f}{n}\right) \frac{S_{x}^{2}}{\bar{Y}} R_{13}^{2}$ & $\begin{array}{l}\left(\frac{1-f}{n}\right)\left(R_{13}^{2} S_{x}^{2}\right. \\
\left.+S_{y}^{2}\left(1-\rho_{y x}^{2}\right)\right)\end{array}$ & $\begin{array}{l}R_{13} \\
=\frac{\bar{Y} C_{x}}{\bar{X} C_{x}+\rho_{y x}}\end{array}$ \\
\hline $\begin{array}{l}t_{14} \\
\quad=\frac{\bar{y}+b(\bar{X}-\bar{x})}{\bar{x} \rho_{y x}+C_{x}}\left(\bar{X} \rho_{y x}\right. \\
\left.+C_{x}\right) \\
\text { Kadilar and Cingi (2006) }\end{array}$ & $\left(\frac{1-f}{n}\right) \frac{S_{x}^{2}}{\bar{Y}} R_{14}^{2}$ & $\begin{array}{l}\left(\frac{1-f}{n}\right)\left(R_{14}^{2} S_{x}^{2}\right. \\
\left.+S_{y}^{2}\left(1-\rho_{y x}^{2}\right)\right)\end{array}$ & $\begin{array}{l}R_{14} \\
=\frac{\bar{Y} \rho_{y x}}{\bar{X} \rho_{y x}+C_{x}}\end{array}$ \\
\hline $\begin{array}{l}t_{15} \\
\quad=\frac{\bar{y}+b(\bar{X}-\bar{x})}{\bar{x} \rho_{y x}+\beta_{2}}\left(\bar{X} \rho_{y x}\right. \\
\left.+\beta_{2}\right) \\
\text { Kadilar and Cingi (2006) }\end{array}$ & $\left(\frac{1-f}{n}\right) \frac{S_{x}^{2}}{\bar{Y}} R_{15}^{2}$ & $\begin{array}{l}\left(\frac{1-f}{n}\right)\left(R_{15}^{2} S_{x}^{2}\right. \\
\left.+S_{y}^{2}\left(1-\rho_{y x}^{2}\right)\right)\end{array}$ & $\begin{array}{l}R_{15} \\
=\frac{\bar{Y} \rho_{y x}}{\bar{X} \rho_{y x}+\beta_{2}}\end{array}$ \\
\hline $\begin{array}{l}\qquad t_{16}=\bar{y}\left(\frac{\bar{X}+M_{d}}{\bar{x}+M_{d}}\right) \\
\text { Subramani and } \\
\text { Kumarapandiyan (2013) }\end{array}$ & $\begin{array}{l}\left(\frac{1-f}{n}\right) \bar{Y}\left(R_{16}^{2} C_{x}^{2}\right. \\
\left.-R_{16} \rho_{y x} C_{x} C_{y}\right)\end{array}$ & $\begin{array}{l}\left(\frac{1-f}{n}\right) \bar{Y}^{2}\left(C_{y}^{2}\right. \\
+R_{16}^{2} C_{x}^{2} \\
\left.-2 R_{16} \rho_{y x} C_{x} C_{y}\right)\end{array}$ & $\begin{array}{l}R_{16} \\
=\frac{\bar{X}}{\bar{X}+M_{d}}\end{array}$ \\
\hline
\end{tabular}




\section{Proposed Estimator}

Following Subramani and Kumarapandiyan (2013), I have proposed an estimator for estimating the population mean when the population mean and population median of the auxiliary variable is known

$$
t_{s}=\bar{y}\left\{\alpha\left(\frac{\bar{X}+M_{d}}{\bar{x}+M_{d}}\right)+(1-\alpha)\left(\frac{\bar{x}+M_{d}}{\bar{X}+M_{d}}\right)\right\}
$$

where $M_{d}$ is the population median of the auxiliary variable X.

To the first degree of approximation, I have obtained the expression of bias and mean squared error (MSE) of the proposed estimator as

$$
B\left(t_{s}\right)=\left(\frac{1-f}{n}\right) \bar{Y}\left\{(1-2 \alpha) \emptyset \rho_{y x} C_{y} C_{x}+\alpha \emptyset^{2} C_{x}^{2}\right\}
$$

and

$$
\operatorname{MSE}\left(t_{s}\right)=\left(\frac{1-f}{n}\right) \bar{Y}\left\{C_{y}^{2}+(1-2 \alpha)^{2} \emptyset^{2} C_{x}^{2}+2(1-2 \alpha) \emptyset \rho_{y x} C_{y} C_{x}\right\}
$$

where $\varnothing=\frac{\bar{X}}{\bar{X}+M_{d}}$.

The $\operatorname{MSE}\left(t_{s}\right)$ will be minimum when

$$
\alpha=\frac{1}{2}\left(1-\frac{\rho_{y x} C_{y}}{\varnothing C_{x}}\right)=\alpha_{0}(\text { say })
$$

By Substituting the minimum value of $\alpha$ in the proposed estimator, one can get the asymptotically optimum estimator (AOE) as

$$
t_{s}(o p t)=\bar{y}\left\{\alpha_{0}\left(\frac{\bar{X}+M_{d}}{\bar{x}+M_{d}}\right)+\left(1-\alpha_{0}\right)\left(\frac{\bar{x}+M_{d}}{\bar{X}+M_{d}}\right)\right\}
$$

Thus, the optimum MSE of $t_{S}$ is

$$
\operatorname{Min} \cdot \operatorname{MSE}\left(t_{s}\right)=\left(\frac{1-f}{n}\right) \bar{Y}\left(1-\rho_{y x}^{2}\right) C_{y}^{2} .
$$

\section{Efficiency Comparison}

For comparison of proposed estimator with the existing estimators, I have derived the conditions for which the proposed estimator is more efficient than the existing modified ratio estimators as

$$
\begin{aligned}
& \operatorname{Min.} M S E\left(t_{s}\right) \leq \operatorname{MSE}\left(t_{i} ; i=1,2,3,4,5\right) \text { if } \rho_{y x} \leq \frac{\theta_{i} C_{x}}{C_{y}} \\
& \operatorname{Min.} \operatorname{MSE}\left(t_{s}\right) \leq \operatorname{MSE}\left(t_{i} ; i=6 \text { to } 15\right) \text { if } R_{i}^{2} S_{x}^{2} \geq 0 \\
& \operatorname{Min.} \operatorname{MSE}\left(t_{s}\right) \leq \operatorname{MSE}\left(t_{16}\right) \text { if } \rho_{y x} \leq \frac{R_{16} C_{x}}{C_{y}} . \\
& \operatorname{Min.} \operatorname{MSE}\left(t_{s}\right) \leq \operatorname{MSE}\left(t_{R}\right) \text { if } \rho_{y x} \leq \frac{C_{x}}{C_{y}}
\end{aligned}
$$

From the above conditions, it is noted that the proposed estimator is more efficient among other discussed estimators if the above conditions holds true. 


\section{Empirical Study}

To demonstrate the performance of the suggested estimator empirically in comparison to other estimators. I have used three natural population data sets. The descriptions of the populations are given below.

Population I: [Source: Murthy (1967)]

Y: Output for 80 factories in a region

$\mathrm{X}$ : Fixed Capital

$N=80 ; n=20 ; \bar{Y}=51.8264 ; \bar{X}=11.2646 ; \rho_{y x}=0.9413 ; S_{y}=$ 18.3569; $C_{y}=0.3542 ; S_{x}=8.4563 ; C_{x}=0.7507 ; \beta_{1}=1.05 ; \beta_{2}=$ $-0.06339 ; M_{d}=7.575$

Population II: [Source: Murthy (1967)]

Y: Output for 80 factories in a region

$X$ : Data on number of workers

$N=80 ; n=20 ; \bar{Y}=51.8264 ; \bar{X}=2.8513 ; \rho_{y x}=0.9150 ; S_{y}=$ 18.3569; $C_{y}=0.3542 ; S_{x}=2.7042 ; C_{x}=0.9484 ; \beta_{1}=0.6978 ; \beta_{2}=$ $1.3005 ; M_{d}=1.48$

Population III: [Source: Cochran (1977)]

Y: Number of persons

$X$ : Number of rooms $N=10 ; n=4 ; \bar{Y}=101.00 ; \bar{X}=58.80 ; \rho_{y x}=0.6515 ; S_{y}=14.6523 ; C_{y}=$ $0.1449 ; S_{x}=7.5339 ; C_{x}=0.1281 ; \beta_{1}=0.5764 ; \beta_{2}=-0.3814 ; M_{d}=58$ Here, I have computed mean squared error (MSE) and the constants of the estimators.

The results are given in the following table.

Table 2: The mean squared errors and constants of the existing and proposed estimators

\begin{tabular}{|c|c|c|c|c|c|c|}
\hline \multirow{2}{*}{ Estimator } & \multicolumn{6}{|c|}{ MSE and Constants } \\
\cline { 2 - 7 } & \multicolumn{2}{|c|}{ Population I } & \multicolumn{2}{c|}{ Population II } & \multicolumn{2}{c|}{ Population III } \\
\cline { 2 - 7 } & MSE & Constants & MSE & Constants & MSE & Constants \\
\hline $\begin{array}{c}t_{1} ; \text { Sisodia and } \\
\text { Dwivedi(1981) }\end{array}$ & 15.25812 & 0.937521 & 17.18812 & 0.750401 & 20.2396 & 0.997826 \\
\hline $\begin{array}{c}t_{2} ; \text { Singh et al } \\
(2004)\end{array}$ & 19.33825 & 1.005659 & 12.84257 & 0.686762 & 20.14213 & 0.990292 \\
\hline $\begin{array}{c}t_{3} ; \text { Yan and Tian } \\
(2010)\end{array}$ & 14.01128 & 0.914735 & 21.36603 & 0.803387 & 20.35575 & 1.006529 \\
\hline $\begin{array}{c}t_{4} ; \text { Singh and } \\
\text { Tailor (2003) }\end{array}$ & 14.45027 & 0.922882 & 17.68491 & 0.757056 & 20.12623 & 0.989041 \\
\hline $\begin{array}{c}t_{5} ; \text { Upadhyaya and } \\
\text { Singh (1999) }\end{array}$ & 19.45917 & 1.007553 & 12.1351 & 0.675254 & 19.45448 & 0.928916 \\
\hline
\end{tabular}


Table 2 (continued):

\begin{tabular}{|c|c|c|c|c|c|c|}
\hline $\begin{array}{c}t_{6} ; \text { Kadilar and } \\
\text { Cingi (2004) }\end{array}$ & 58.20263 & 4.60082 & 92.65628 & 18.17641 & 43.70438 & 1.719388 \\
\hline $\begin{array}{c}t_{7} ; \text { Kadilar and } \\
\text { Cingi (2004) }\end{array}$ & 51.3313 & 4.313367 & 53.07362 & 13.6396 & 43.59507 & 1.71565 \\
\hline $\begin{array}{c}t_{8} ; \text { Kadilar and } \\
\text { Cingi (2004) }\end{array}$ & 58.84691 & 4.626857 & 44.78744 & 12.48287 & 43.21808 & 1.702697 \\
\hline $\begin{array}{c}t_{9} ; \text { Kadilar and } \\
\text { Cingi (2004) }\end{array}$ & 4.130342 & 1.001632 & 2.317192 & 0.974223 & 26.33849 & 0.95739 \\
\hline $\begin{array}{c}t_{10} ; \text { Yan and Tian } \\
(2010)\end{array}$ & 48.93561 & 4.208533 & 60.5325 & 14.60269 & 44.0341 & 1.730613 \\
\hline $\begin{array}{c}t_{11} ; \text { Yan and Tian } \\
(2010)\end{array}$ & 58.81598 & 4.625611 & 35.18871 & 10.99178 & 45.04985 & 1.764745 \\
\hline $\begin{array}{c}t_{12} ; \text { Kadilar and } \\
\text { Cingi (2006) }\end{array}$ & 49.78532 & 4.246012 & 53.98248 & 13.76056 & 43.15575 & 1.700546 \\
\hline $\begin{array}{c}t_{13} ; \text { Kadilar and } \\
\text { Cingi (2006) }\end{array}$ & 47.40103 & 4.139986 & 52.63652 & 13.58105 & 39.85643 & 1.58251 \\
\hline $\begin{array}{c}t_{14} ; \text { Kadilar and } \\
\text { Cingi (2006) }\end{array}$ & 50.94479 & 4.296627 & 50.78761 & 13.33051 & 43.53689 & 1.713657 \\
\hline $\begin{array}{c}t_{15} ; \text { Kadilar and } \\
\text { Cingi (2006) }\end{array}$ & 58.88745 & 4.628491 & 42.40512 & 12.12991 & 42.96371 & 1.693901 \\
\hline $\begin{array}{c}t_{16} ; \text { Subramani and } \\
\text { Kumarapandiyan } \\
(2013)\end{array}$ & & & & & & \\
\hline $\begin{array}{c}\boldsymbol{t}_{\boldsymbol{s}} ; \text { (Proposed } \\
\text { Estimator) }\end{array}$ & $\mathbf{0 . 0 6 8 0 2 8}$ & $\mathbf{0 . 5 9 7 9 2 1}$ & $\mathbf{0 . 0 0 6 2 2 6}$ & $\mathbf{0 . 6 5 8 3 0 1}$ & $\mathbf{6 . 2 6 7 0 6 3}$ & $\mathbf{0 . 5 0 3 4 2 5}$ \\
\hline
\end{tabular}

From the above table, it is envisaged that the proposed optimum estimator is more efficient than other existing estimator mentioned in Table 1 in terms of less mean squared error (MSE).

\section{Conclusion}

In this paper, I have proposed a modified estimator based on simple random sampling without replacement by using auxiliary variable, under the situation when population mean and median of the auxiliary variable is known. It is found that the performance of the proposed estimator in terms of bias and mean squared error is more efficient than all other existing estimator for certain known population parameters of auxiliary variable. The above results are supported theoretically and empirically by three natural populations. 


\section{Acknowledgements}

Author wishes to thank the learned referees for their critical and constructive comments regarding improvement of the paper.

\section{References}

1. Cochran, W. G. (1940). The estimation of the yields of the cereal experiments by sampling for the ratio of grain to total produce. Journal of Agricultural Science, 30: 262-275, http://dx.doi.org/10.1017/S0021859600048012.

2. Cochran, W. G. (1977). Sampling Techniques, Third Edition, Wiley Eastern Limited, PMCid:PMC1607049.

3. Kadilar, C. and Cingi, H. (2004). Ratio estimators in simple random sampling. Applied Mathematics and Computation, 151: 893-902, http://dx.doi.org/10.1016/S0096-3003(03)00803-8.

4. Kadilar, C. and Cingi, H. (2006). An Improvement in Estimating the Population mean by using the Correlation Coefficient. Hacettepe Journal of Mathematics and Statistics, 35 (1): 103-109.

5. Koyuncu, N. and Kadilar, C. (2009). Efficient Estimators for the Population mean. Hacettepe Journal of Mathematics and Statistics, 38(2): 217-225.

6. Murthy, M.N. (1967). Sampling theory and methods, Statistical Publishing Society, Calcutta, India.

7. Prasad, B. (1989). Some improved ratio type estimators of population mean and ratio in finite population sample surveys. Communications in Statistics: Theory and Methods, 18: 379-392, http://dx.doi.org/10.1080/03610928908829905.

8. Rao, T.J. (1991). On certain methods of improving ratio and regression estimators. Communications in Statistics: Theory and Methods, 20(10): 3325-3340, http://dx.doi.org/10.1080/03610929108830705.

9. Robson, D. S. (1957). Application of multivariate polykays to the theory of unbiased ratio type estimation. Journal of American Statistical Association, 52: 411-422, http://dx.doi.org/10.1080/01621459.1957.10501407.

10. Singh, D. and Chaudhary, F.S. (1986). Theory and Analysis of Sample Survey Designs, New Age International Publisher. 
11. Singh, G.N. (2003). On the improvement of product method of estimation in sample surveys. Journal of the Indian Society of Agricultural Statistics, 56 (3): 267-265.

12. Singh, H.P. and Tailor, R. (2003). Use of known correlation coefficient in estimating the finite population means. Statistics in Transition - new series, 6 (4): 555-560.

13. Singh, H.P., Tailor, R., Tailor, R. and Kakran, M.S. (2004). An Improved Estimator of population means using Power transformation. Journal of the Indian Society of Agricultural Statistics, 58(2): 223-230.

14. Singh, H.P. and Tailor, R. (2005). Estimation of finite population mean with known coefficient of variation of an auxiliary. STATISTICA, anno LXV(3): 301-31.

15. Sisodia, B.V.S. and Dwivedi, V.K. (1981). A modified ratio estimator using coefficient of variation of auxiliary variable. Journal of the Indian Society of Agricultural Statistics, 33(1): 13-18.

16. Upadhyaya, L.N. and Singh, H.P. (1999). Use of transformed auxiliary variable in estimating the finite population means. Biometrical Journal, 41(5): 627-636, http://dx.doi.org/10.1002/(SICI)15214036(199909)41:5<627::AID-BIMJ627>3.0.CO;2-W.

17. Yan, Z. and Tian, B. (2010). Ratio Method to the Mean Estimation Using Coefficient of Skewness of Auxiliary Variable. ICICA, Part II, CCIS 106: 103-110, http://dx.doi.org/10.1007/978-3-642-16339-5_14. 